Review

\title{
Oxidative stress, genomic features and DNA repair in frail elderly: A systematic review
}

\author{
María Sánchez-Flores a,b,1, Diego Marcos-Pérez ${ }^{a, b, 1}$, Solange Costac ${ }^{\mathrm{a}}$, João Paulo Teixeira ${ }^{\mathrm{c}}$, \\ Stefano Bonassi ${ }^{\mathrm{d}, \mathrm{e}}$, Eduardo Pásaro ${ }^{\mathrm{a}}$, Blanca Laffon ${ }^{\mathrm{a}, *, 2}$, Vanessa Valdiglesias ${ }^{\mathrm{a}, c, 2}$ \\ a Universidade da Coruña, DICOMOSA Group, Department of Psychology, Area of Psychobiology, Edificio de Servicios Centrales de Investigación, Campus \\ Elviña s/n, 15071, A Coruña, Spain \\ b Universidade da Coruña, Department of Cell and Molecular Biology, A Coruña, Spain

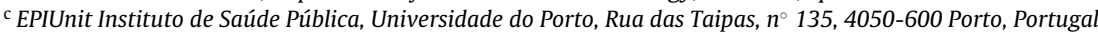 \\ d Department of Human Sciences and Quality of Life Promotion, San Raffaele University, Rome, Italy \\ e Unit of Clinical and Molecular Epidemiology, IRCCS San Raffaele Pisana, Rome, Italy
}

\section{A R T I C L E I N F O}

\section{Article history:}

Received 9 April 2017

Received in revised form 28 April 2017

Accepted 2 May 2017

Available online 6 May 2017

\section{Keywords:}

Cellular biomarkers

DNA repair

Frailty

Genomic instability

Elderly

Oxidative stress

\begin{abstract}
A B S T R A C T
Frailty is an emerging geriatric syndrome characterized by higher vulnerability to stressors, with an increased risk of adverse health outcomes such as mortality, morbidity, disability, hospitalization, and institutionalization. Although it is generally recognized to have a biological basis, no particular biological trait has been consistently associated to frailty status so far. In this work, epidemiological studies evaluating association of frailty status with alterations at cellular level - namely oxidative stress, genomic instability and DNA damage and repair biomarkers - were revised and compared. A total of 25 studies fulfilled inclusion/exclusion criteria and, consequently, were included in the review. Variations of oxidative stress biomarkers were often associated to frailty status in older people. On the contrary, genomic instability seems not to be linked to frailty. The only study which addressed the possible relationship between DNA repair modulations and frailty status also failed in finding association. Despite the large number of cellular alterations known to be associated with frailty, studies on this issue are still very scarce and limited to some of the possible cellular targets. The established link between DNA repair, genomic instability, and age and age-related disorders, encourage deeper investigations on this line.
\end{abstract}

(c) 2017 Elsevier B.V. All rights reserved.

\section{Contents}

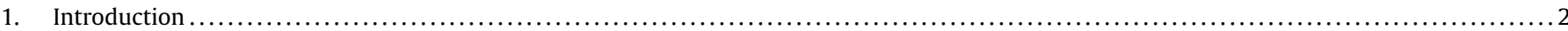

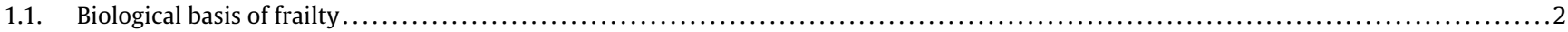

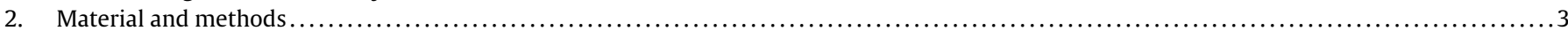

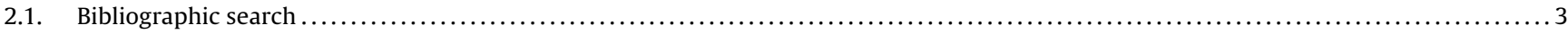

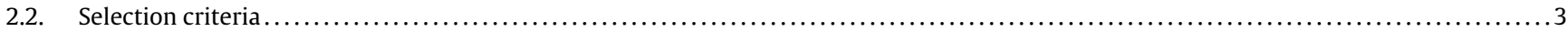

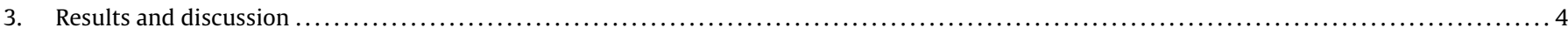

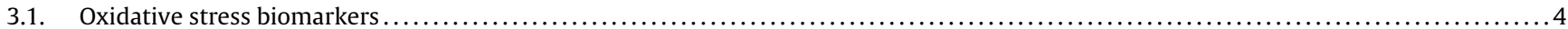

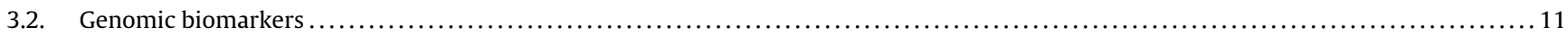

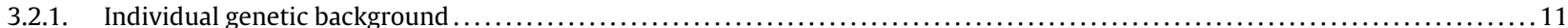

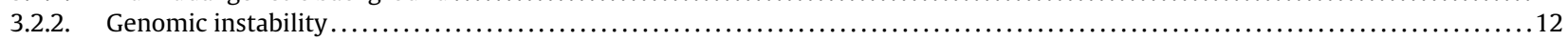

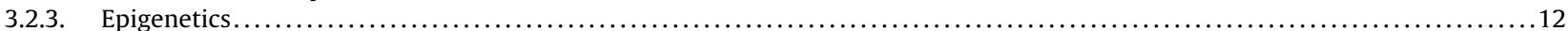

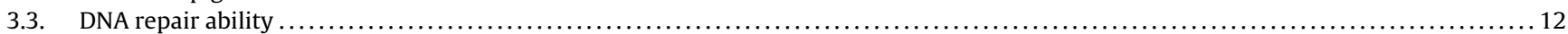

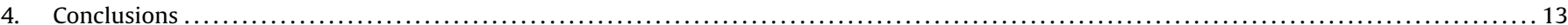

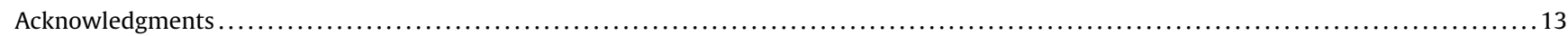

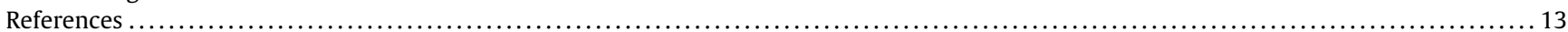

\footnotetext{
* Corresponding author.

E-mail address: blaffon@udc.es (B. Laffon).

1 These authors contributed equally to this work.

2 These authors contributed equally to the senior authorship of this manuscript.
} 


\section{Introduction}

The concept of frailty is getting more and more attention as a new and more accurate way to define biological age as well as to identify vulnerability in elderly. Frailty is an important geriatric syndrome that represents a risk factor for those over 65 years old, and its prevalence increases with advancing age (Topinková, 2008). Identifying frail people as early as possible is socially and economically crucial since evidence from different studies suggests that frailty status, particularly at its very earliest stages, might present characteristics of reversibility (Espinoza et al., 2012; Gill et al., 2006; Roland et al., 2014). Thus, frailty criteria should be used by geriatricians and healthcare professionals in order to provide a proper and personalized care to the older individual. But currently, there is no consensus in an international official definition of frailty, being considered as a condition, syndrome or status depending on the author or publication.

Currently, there are several tools that are used for screening frailty in older adults. The two most well-known instruments to identify frailty are the phenotypic model proposed by Fried et al. (2001), and the deficit accumulation model developed by Rockwood, Mitnitski and collaborators (Mitnitski et al., 2001; Rockwood et al., 2005). The model proposed by Fried et al. is based on five criteria that include shrinking or unintentional weight loss, muscular weakness, self-reported exhaustion, slow walk and low physical activity level. Those individuals with three or more of those criteria are considered frail, while those with one or two are considered pre-frail, and those individuals who do not show any of those are considered non-frail or robust (Fried et al., 2001).The cumulative model proposed by Rockwood and Mitnitski (Mitnitski et al., 2001; Rockwood et al., 2005), also called frailty index (FI), is calculated as the ratio between the number of deficits the individual presents divided by the total of deficits considered in the computation. The deficits evaluated include physical criteria, neurological examinations, psychological symptoms, and clinical laboratory values, among others.

In addition to these models, there are many other screening tools to identify frailty, including a number of variants of the original criteria. For instance, Montesanto scale is a population-specific survey consisting of a cluster analysis based on three phenotypic parameters [Mini Mental State Examination (MMSE) (Folstein et al., 1975), hand grip strength and Geriatric Depression Scale (GDS) (Sheikh and Yesavage, 1986)], adjusted by physical and clinical parameters (height, weight, knee-to-floor height and waist and hip circumferences, functional activity and health status) (Montesanto et al., 2010). Hospital admission risk profile (HARP) is another different instrument for stratifying older patients at the time of hospital admission, according to their risk of developing new disabilities in activities of daily living (ADL) following acute medical illness and hospitalization (Sager et al., 1996). This index is based on demographic information (age, gender, mental status, living arrangement, race), ability to perform six ADL (bathing, dressing, transferring, walking, toileting, and eating) and seven instrumental activities of daily living (IADL) (managing finances, taking medications, telephoning, shopping, using transportation, preparing meals, and doing housework) two weeks before admission. An abbreviated MMSE (range 0-21) is also obtained during the admission interview.

In a recent review the existence of more than 260 different versions of the frailty phenotype published in the literature was reported. And even though all of them might potentially identify frailty, the modifications introduced in the original phenotype criteria had important impact on its classification and predictive ability (Theou et al., 2015).This observation was later confirmed by Dent et al. (2016), who published another complete review collecting all the frailty measurements employed to date to identify this syndrome, and also pointed out that many frailty measurements were modified somewhat from their original validated version, and that this could eventually have a striking impact on frailty classification. After reviewing a total of 422 studies on frailty and reporting 29 different frailty instruments, Dent et al. (2016) concluded that there is not a single optimal frailty assessment criteria among those proposed so far, and claimed the need of unifying criteria, possibly through the release of guidelines, to establish a standard measurement criteria for frailty, which will make easier the identification of frail subjects in clinical practice, and will make the different studies comparable. This would also help to know the actual frailty prevalence worldwide.

Still, despite the high number of available instruments to measure frailty and their different basis and criteria, it seems that the predictive value of frailty for negative health outcomes, including falls, hospitalizations, disability, institutionalization, and mortality, is consistently confirmed for most assessment tools, target populations, and settings (Clegg et al., 2013; Fried et al., 2009; Theou et al., 2013).

Despite all the efforts focused on establishing a common definition and a more accurate measurement of frailty, the criteria proposed by Fried and collaborators is still the tool most frequently employed to identify frail individuals in clinical practice and research. The working definition of frailty established according to Fried's criteria, however, it is only based on physical symptoms and signs. It neglects other potentially important components of the syndrome such as mood, cognition, sensory impairments, and socioeconomic aspects of older adults' lives (Abellan van Kan et al., 2008; Zaslavsky et al., 2013). Moreover, no biological modifications are considered. Numerous researchers have argued that frailty is a multidimensional and multisystem process that cannot be comprehensively captured by applying physical criteria only (Dent et al., 2016; Zaslavsky et al., 2013).

Consequently, in the last years, more and more authors emphasized the limitations of classifying frail subjects only according to clinical parameters, highlighting the need of other markers of frailty at different levels, and even considering separately physical and cognitive frailty (Dulac and Aubertin-Leheudre, 2016; Kelaiditi et al., 2013; Woods et al., 2013).

\subsection{Biological basis of frailty}

Although, frailty was initially linked to physical decline and considered as synonymous of disability or comorbidity, currently it is becoming recognized as a distinct clinical syndrome with a biological basis (Lang et al., 2009). Indeed, frailty is commonly accepted to have a strong biological component that results from cumulative cellular damage over the life-course (Dent et al., 2016). As people age, many systems and processes can be modified (reviewed in Fielding, 2015). Similarly, a number of physiological processes/functions have been demonstrated to be altered in frail subjects (reviewed in Walston, 2004).

However, the specific pathophysiological changes involved in frailty aetiology remain undefined. This is mainly due to the fact that no single system impairment characterizes frailty. Instead, it seems that an intertwined network of biological anomalies at different levels is likely to be part of the pathophysiological chain of events leading to frailty (Zaslavsky et al., 2013). In 2009, Lang et al. already highlighted the importance of improving our understanding on the complex biological factors leading to age-related muscle loss (sarcopenia, a typical clinical sign in frail subjects), beyond those attributa to a simple decrease in physical activity and to deleterious chronic undernutrition.

All the processes or physiological functions known to be altered in frail patients can be grouped into three different dimensions, according to the organismic level affected (Fig. 1). At the cellu- 


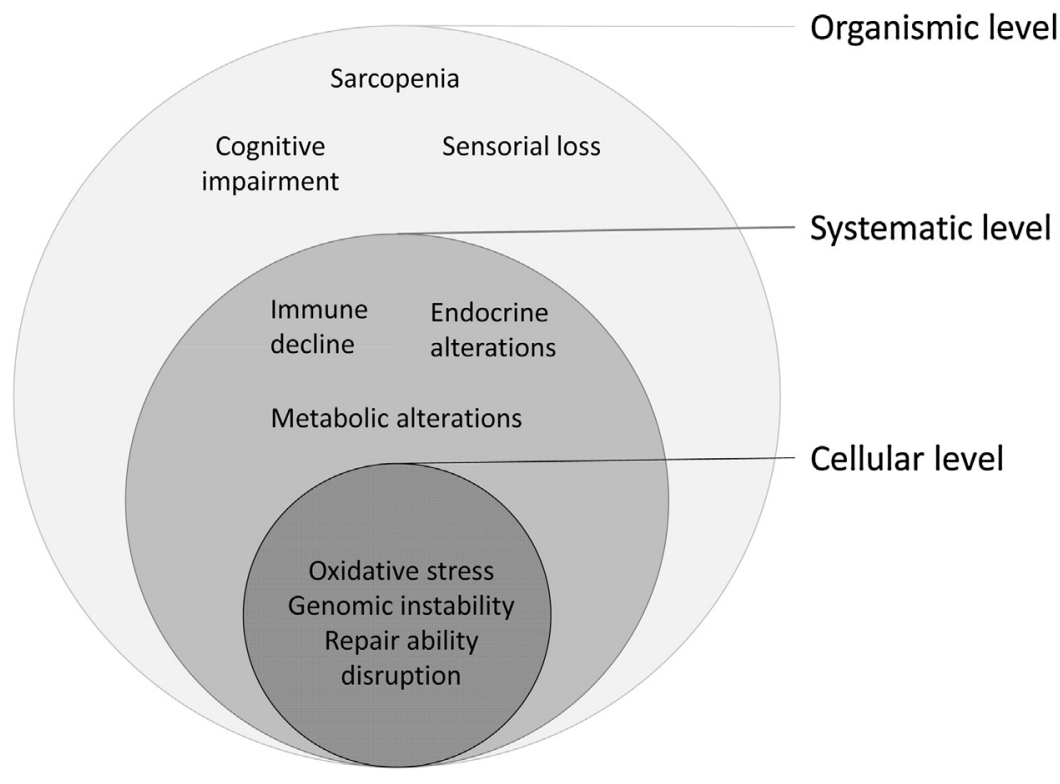

Fig. 1. Biological basis of frailty.

lar level, frailty status has been linked to deficiencies in cellular repair ability and consequent DNA damage accumulation (Dent et al., 2016). The biological consequences of increased levels of damage can be wide ranging, including altered gene expression, genomic instability, mutations, loss of cell division potential, cell death, impaired intercellular communication, tissue disorganization, organ dysfunctions, and increased vulnerability to stress and other sources of disturbance (Rattan, 2006). At the systematic level, more and more evidence suggests that frailty-associated physiological dysregulation involves multi-organ systems, including the musculoskeletal, immune, endocrine, hematologic, and cardiovascular systems (Fried et al., 2009; Li et al., 2011). Finally, as more systems show abnormal function, frailty severity increases, and all these dysregulations, although initially silent, become physically evident, affecting the whole organism, showing up as the clinical signs of frailty: muscle mass loss, cognitive impairment and sensorial loss, among others.

Recent research efforts have helped to better define the clinical and physiological characteristics of frailty (Lang et al., 2009; Sieber, 2017). The use of biomarkers to identify frail subjects not only would be a more precise and objective method for frailty identification, but also would allow to compare epidemiological studies drawing suitable conclusions from them. However, and despite these last evidences on the biological basis of frailty, up to now no biological feature has been validated to be used as a useful biomarker to identify frailty status. In this work, all clinical/epidemiological studies conducted in older frail adults, evaluating any alteration at the cellular level - including biomarkers of oxidative stress, genomic alterations and DNA repair -were reviewed, in order to identify parameters that could be associated with frailty condition and consequently be proposed as biomarkers of frailty.

\section{Material and methods}

\subsection{Bibliographic search}

The identification and selection of studies to be included in the review was carried out through an extensive literature search using the PubMed database (National Library of Medicine, National
Institutes of Health, Bethesda, MD, USA; http://www.ncbi.nih.gov/ PubMed), and was updated to March 2016.

The search strategy developed comprised two terms that were intersected using the Boolean term "AND". The first one included descriptors related to frailty ('frail', 'frailty' or 'frailty elderly'), and the second one included descriptors related to biomarkers at the cellular level ('cellular damage', 'DNA', 'genomic', 'oxidative', or 'DNA repair'). All searches were focused on in title or abstract.

\subsection{Selection criteria}

Eligible studies to be included in the review were all studies conducted in humans, written in English or Spanish, and focused on populations of older adults (mean age $\geq 60$ years old). Study individuals must have been classified according their frailty status following any of the currently validated criteria for frailty identification. Moreover, any cellular or molecular biomarkers must have been evaluated using any methods. In particular, the studies selected for this review can be classified within the following subgroups:

- Those evaluating oxidative stress biomarkers in frail and non-frail older adults

- Those evaluating genomic biomarkers in frail and non-frail older adults

- Those evaluating DNA repair ability in frail and non-frail older adults

Studies not considering frailty but ageing, reviews, studies carried out in animals, and articles not written in English or Spanish were excluded. Finally, studies using criteria not validated to identify frail individuals, or employing frailty as a confounder instead of outcome, were also not included in this review. Whenever the same group of authors published papers on the same group of patients, only the most recent or most complete report was considered.

Two hundred and sixty-six citations (after excluding duplicates) were initially obtained and manually reviewed (Fig. 2). Among them, 75 resulted eligible after an initial revision of abstracts. The whole publications of all these studies were fully reviewed, finding 21 studies that fulfilled the selection criteria. Other five additional 
Pub Med Search

n= 266 (excluding duplicates)

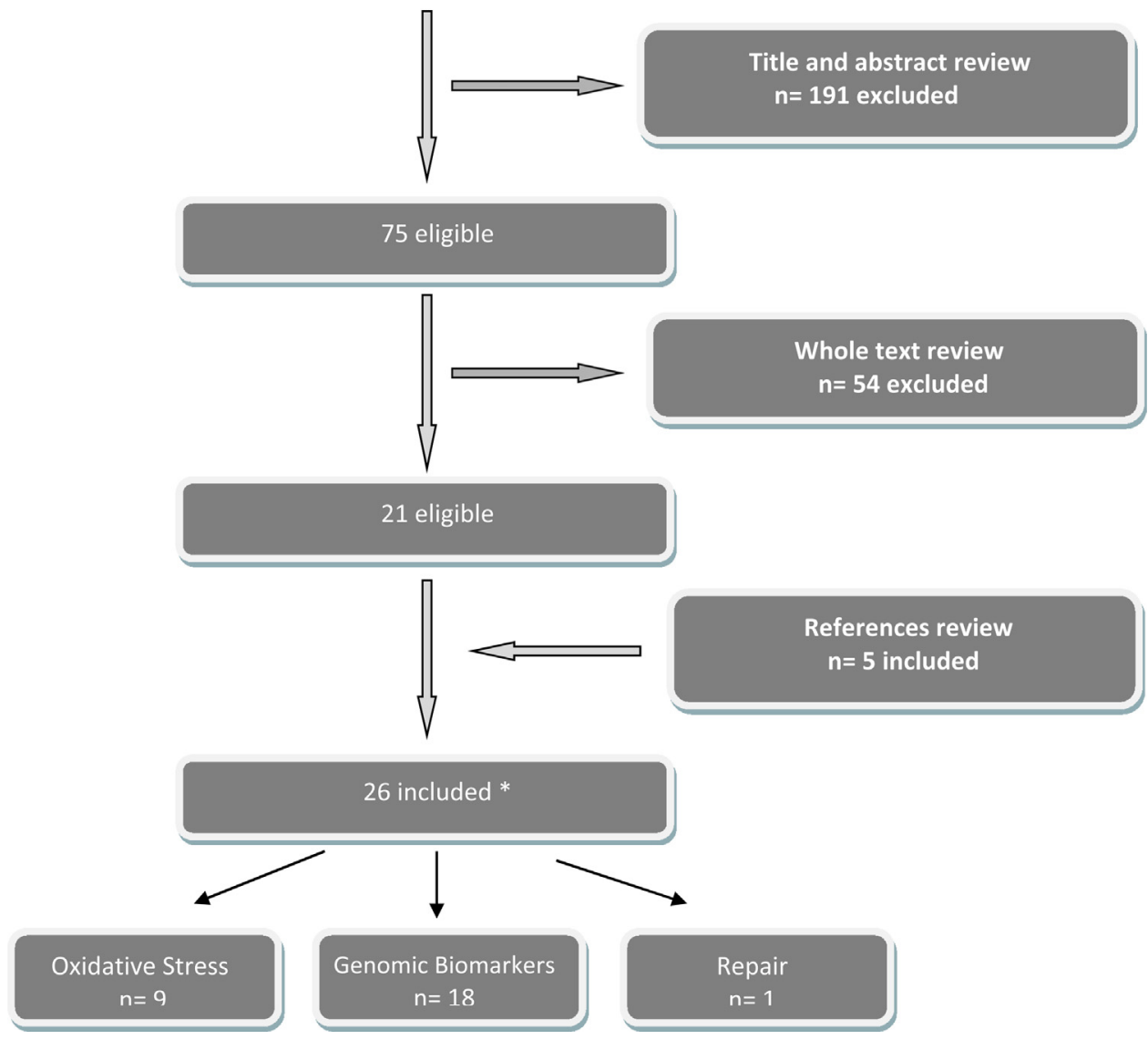

Fig. 2. Flow chart of systematic review. ${ }^{*}$ One paper (Collerton et al., 2012) addressed the three aspects considered in this systematic review.

publications were identified and included in the revision after reviewing the references section of published articles.

\section{Results and discussion}

As a result of the searching process, a total of 26 studies published from 2008 to 2017 suited inclusion/exclusion criteria and, consequently, were included in this review. Among them, 8 evaluated oxidative stress biomarkers and 17 genomic biomarkers. Besides, one study (Collerton et al., 2012) evaluated oxidative stress and genomic biomarkers, plus DNA repair ability on the same population of older adults. All studies but one (Pereira et al., 2016) were written in English. According to the affiliation of the first author, these studies were mostly conducted in Europe (54\%), USA (27\%), and Asia (15\%). The number of individuals analyzed per study ranged from 15 to 5275 , with a mean sample size of 499 per group. Four papers only (15.4\%) included less than 100 individuals, 73\% included more than 300 , and in eight out of 26 reviewed studies (30.8\%) the sample population size was larger than 1000 subjects, confirming the robustness of the data evaluated in this review. The mean age of the studied individuals ranged from 61.9 to 99 years per group, with a total mean age of 66.8 years. Considering all studies in which patients were classified by frailty, the mean age in frail groups was 78.1 years, in pre-frail groups76.4 years, and in non-frail groups 74.4 years.
Regarding the criteria employed to identify frail people, almost all studies (24 out of 26) employed either Fried's or Rockwood's criteria (FI), or even both of them. Among these studies, the great majority (67\%) employed Fried's criteria for frailty identification, and only in two studies a modified version was used instead of the original one. Five out of 24 studies (21\%) used FI to identify frail subjects, and three out of 24 studies (13\%) employed both of them. However, the number of items analyzed in studies employing FI was always different among studies, ranging from 17 to 40 . The two remaining studies not using Fried's or Rockwood's criteria were Bellizzi et al. (2012) and Pereira et al. (2016), which employed the Montesanto et al. (2010) method, and the Hospital Admission Risk Profile (HARP) together with FI, respectively.

All studies included in this review are presented in tables (Tables 1 and 2) and described in the following sections according to the type of parameter evaluated. Although performing a metaanalysis of these studies was initially considered, it was not possible to carry it out due to the high variability in the outcomes addressed together with the limited number of studies assessing the same outcome.

\subsection{Oxidative stress biomarkers}

Reactive oxygen species (ROS) are free radicals formed during the cellular metabolism. Generally, these ROS are not harmful for the cell since they are neutralized by cellular antioxidant systems, 
Table 1

Studies assessing oxidative stress biomarkers.

\begin{tabular}{|c|c|c|c|c|c|c|c|}
\hline Study & $\begin{array}{l}\text { Total population } \\
\text { (mean age } \pm \text { SD) }\end{array}$ & $\begin{array}{l}\text { Case population (mean } \\
\text { age } \pm \text { SD) }\end{array}$ & $\begin{array}{l}\text { Control population } \\
\text { (mean age } \pm \text { SD) }\end{array}$ & Frailty criteria & Outcomes & Assay method & Results \\
\hline Ble et al. (2006) & $\begin{array}{l}\mathrm{n}=827(73.6 \pm 6.4) 446 \\
\text { females, } 381 \text { males }\end{array}$ & Frail $n=54$ & $\begin{array}{l}\text { Pre-frail } n=313 \\
\text { non-frail } n=460\end{array}$ & Fried et al. (2001) & Plasma Vit-E levels & Reverse-phase HPLC & $\begin{array}{l}\text { Levels of vitamin E decreased } \\
\text { gradually from the non-frail to } \\
\text { the frail group }\end{array}$ \\
\hline Collerton et al. (2012) & $\begin{array}{l}\mathrm{n}=552(85) 332 \\
\text { females, } 220 \text { males }\end{array}$ & $\begin{array}{l}\text { Frail } n=119(85) 92 \\
\text { females, } 27 \text { males }\end{array}$ & $\begin{array}{l}\text { Pre-frail } n=333(85) \\
193 \text { females, } 140 \\
\text { males; } \\
\text { Non-frail } n=100(85) \\
47 \text { females, } 53 \text { males }\end{array}$ & $\begin{array}{l}\text { Fried et al. (2001) } \\
\text { FI ( } 40 \text { deficits) }\end{array}$ & $\begin{array}{l}\text { Plasma lipid } \\
\text { peroxidation: } \\
\text { isoprostanes iPF2 } \\
\text { alpha-III and iPF2 } \\
\text { alpha-VI }\end{array}$ & LC/MS/MS & $\begin{array}{l}\text { No association lipid } \\
\text { peroxidation with frailty }\end{array}$ \\
\hline \multirow[t]{2}{*}{ Ingles et al. (2014) } & \multirow[t]{2}{*}{$\mathrm{n}=742$} & \multirow[t]{2}{*}{$\begin{array}{l}\text { Frail } \mathrm{n}=54(78.8 \pm 6.0) \\
36 \text { females, } 18 \text { males }\end{array}$} & $\begin{array}{l}\text { Pre-frail } \mathrm{n}=278 \\
\text { ( } 73.8 \pm 4.7) 160 \\
\text { females, } 118 \text { males; }\end{array}$ & \multirow[t]{2}{*}{ Fried et al. (2001) } & $\begin{array}{l}\text { Plasma lipid } \\
\text { peroxidation: MDA }\end{array}$ & $\begin{array}{l}\text { HPLC } \\
\text { Western blot }\end{array}$ & $\begin{array}{l}\text { Frail people had higher MDA } \\
\text { levels than non-frail subjects. }\end{array}$ \\
\hline & & & $\begin{array}{l}\mathrm{n}=410 \text { non-frail } \\
(72.4 \pm 4.2) 237 \\
\text { females, } 173 \text { males }\end{array}$ & & $\begin{array}{l}\text { Plasma protein } \\
\text { carbonylation }\end{array}$ & & $\begin{array}{l}\text { Frail people had higher levels } \\
\text { of protein carbonylation than } \\
\text { non-frail subjects }\end{array}$ \\
\hline Liu et al. (2016) & $\mathrm{n}=1919$ & $\begin{array}{l}\text { Frail } n=142(77 \pm 6) 77 \\
\text { females, } 65 \text { males }\end{array}$ & $\begin{array}{l}\text { Pre-frail } n=864 \\
(72 \pm 7) 495 \text { females, } \\
369 \text { males; } \\
\text { non-frail } n=913 \\
(69 \pm 6) 463 \text { females, } \\
450 \text { males }\end{array}$ & Fried et al. (2001) & $\begin{array}{l}\text { Plasma LpPLA2 activity } \\
\text { and mass, serum } \\
\text { ICAM-1 and MCP-1, } \\
\text { and urine8-epi- } \\
\text { PGF } \alpha \text { isoprostanes }\end{array}$ & ELISA & $\begin{array}{l}\text { Frailty was individually } \\
\text { associated withisoprostanes, } \\
\text { LpPLA2 mass, ICAM-1,and } \\
\text { MCP-1. } \\
\text { Pre-frailty was individually } \\
\text { associated with elevated levels } \\
\text { of LpPLA2 activity, ICAM- } 1 \text {, } \\
\text { and MCP-1 } \\
\text { In those individuals } \geq 70 \text { years } \\
\text { old, associations between } \\
\text { isoprostanes and frailty, and } \\
\text { betweenpre-frailty and MCP-1 } \\
\text { were no longer significant }\end{array}$ \\
\hline \multirow[t]{4}{*}{ Namioka et al. (2016) } & \multirow[t]{4}{*}{$\mathrm{n}=140$} & \multirow[t]{4}{*}{$\begin{array}{l}\text { Frail } \mathrm{n}=34(82.3 \pm 6.1) \\
23 \text { females, } 11 \text { males }\end{array}$} & \multirow{4}{*}{$\begin{array}{l}\text { Pre-frail } \mathrm{n}=62 \\
(80.5 \pm 4.9) 40 \text { females, } \\
22 \text { males; } \\
\text { Non-frail } \mathrm{n}=44 \\
(78.2 \pm 6.0) 19 \\
\text { females, } 25 \text { males }\end{array}$} & \multirow[t]{4}{*}{ Fried et al. (2001) } & Plasma dROM & $\begin{array}{l}\text { Free radical analyzer } \\
\text { system } \\
\text { (spectrophotometry) }\end{array}$ & $\begin{array}{l}\text { dROM levels:frail > pre- } \\
\text { frail > non-frail } \\
\text { individuals }\end{array}$ \\
\hline & & & & & Plasma BAP & $\begin{array}{l}\text { Free radical analyzer } \\
\text { system } \\
\text { (spectrophotometry) }\end{array}$ & $\begin{array}{l}\text { BAP levels were significantly } \\
\text { lower in the frail group than in } \\
\text { the non-frail group }\end{array}$ \\
\hline & & & & & $\begin{array}{l}\text { Endogenous plasma } \\
\text { anti-oxidants: albumin, } \\
\text { bilirubin, uric acid }\end{array}$ & $\begin{array}{l}\text { Bromocresol purple } \\
\text { staining, vanadic acid } \\
\text { oxidation, and uricase } \\
\text { assay, respectively }\end{array}$ & $\begin{array}{l}\text { Bilirubin levels were } \\
\text { significantly lower in the frail } \\
\text { group than in the non-frail } \\
\text { group.No association albumin } \\
\text { or uric acid with frailty }\end{array}$ \\
\hline & & & & & $\begin{array}{l}\text { Urinary 8-OHdG and } \\
\text { 8-epiPGF2 } \alpha\end{array}$ & $\begin{array}{l}\text { HPLC and enzyme } \\
\text { immunoassay, } \\
\text { respectively }\end{array}$ & $\begin{array}{l}\text { Urinary excretions of 8-OHdG } \\
\text { and 8-epiPGF } 2 \alpha \text { were } \\
\text { significantly higher in the frail } \\
\text { and pre-frail groups than inthe } \\
\text { non-frail group }\end{array}$ \\
\hline
\end{tabular}


Table 1 (Continued)

\begin{tabular}{|c|c|c|c|c|c|c|c|}
\hline Study & $\begin{array}{l}\text { Total population } \\
\text { (mean age } \pm \text { SD) }\end{array}$ & $\begin{array}{l}\text { Case population (mean } \\
\text { age } \pm \text { SD) }\end{array}$ & $\begin{array}{l}\text { Control population } \\
\text { (mean age } \pm \text { SD) }\end{array}$ & Frailty criteria & Outcomes & Assay method & Results \\
\hline \multirow[t]{2}{*}{ Pereira et al. (2016) } & \multirow[t]{2}{*}{$\mathrm{n}=15(88 \pm 9.78)$} & $\begin{array}{l}\text { High risk of frailty } \\
(\text { HARP) } n=7\end{array}$ & $\begin{array}{l}\text { Intermediate or low } \\
\text { risk of frailty } \\
\text { (HARP)n }=7\end{array}$ & \multirow[t]{2}{*}{$\begin{array}{l}\text { HARP } \\
\text { FI }\end{array}$} & $\begin{array}{l}\text { Lymphocyte } \\
\text { membranelipid } \\
\text { peroxidation: } \\
\text { conjugated dienes and } \\
\text { trienes }\end{array}$ & UV spectrophotometry & $\begin{array}{l}\text { Elderly patients with a higher } \\
\text { degree of frailty had } \\
\text { significantly higher level of } \\
\text { dienes in both frailty scales, } \\
\text { and significantly higher level of } \\
\text { trienes only in FI }\end{array}$ \\
\hline & & $\begin{array}{l}\text { Intermediate or severe } \\
\text { frailty }(\mathrm{FI}) \mathrm{n}=12\end{array}$ & $\begin{array}{l}\text { Non-frail/mild } \\
\text { frailty(FI)n=3 }\end{array}$ & & $\begin{array}{l}\text { Plasma protein } \\
\text { oxidation: MDA } \\
\text { protein adducts }\end{array}$ & TBARS test & $\begin{array}{l}\text { Elderly patients with a higher } \\
\text { degree of frailty (HARP) } \\
\text { presented significantly higher } \\
\text { levels of MDA than those with } \\
\text { milder levels of frailty }\end{array}$ \\
\hline Saum et al. (2015) & $\mathrm{n}=2518$ & $\begin{array}{l}\text { Frail } \mathrm{n}=210 \\
(73.7 \pm 6.0) 136 \\
\text { females, } 74 \text { males }\end{array}$ & $\begin{array}{l}\text { Pre-frail } \mathrm{n}=1463 \\
(70.3 \pm 6.2) 820 \\
\text { females, } 643 \text { males; } \\
\text { non-frail } \mathrm{n}=845 \\
(67.8 \pm 5.8) 367 \\
\text { females, } 478 \text { males }\end{array}$ & Fried et al. (2001) & Plasma dROM & Spectrophotometry & $\begin{array}{l}\text { Correlation between dROM } \\
\text { levels andfrailty was } \\
\text { statistically significant, but } \\
\text { attenuated after adjustment } \\
\text { with multiple covariates. } \\
\text { An inverse statistically } \\
\text { significant association with } \\
\text { frailty was observed for TTL. }\end{array}$ \\
\hline \multirow[t]{4}{*}{ Serviddio et al. (2009) } & \multirow[t]{4}{*}{$\begin{array}{l}\mathrm{n}=62(76.7 \pm 5.1) \\
23 \text { females,39 males }\end{array}$} & \multirow[t]{4}{*}{ Frail $n=43$} & \multirow[t]{4}{*}{$\mathrm{n}=19$ non-frail } & \multirow[t]{4}{*}{ Fried et al. (2001) } & $\begin{array}{l}\text { Plasma BAP } \\
\text { GSSG }\end{array}$ & $\begin{array}{l}\text { Alkaline hydrolysis of } \\
\mathrm{N} \text {-ethylmaleimide }\end{array}$ & $\begin{array}{l}\text { No association BAP with frailty } \\
\text { A significant increase in the } \\
\text { GSSG was observed in frail } \\
\text { patients when compared to } \\
\text { non-frail }\end{array}$ \\
\hline & & & & & $\begin{array}{l}\text { Whole bloodtotal GSH } \\
\text { level }\end{array}$ & Spectrophotometry & $\begin{array}{l}\text { No association GSH level with } \\
\text { frailty } \\
\text { Frail patients exhibited an } \\
\text { increase in the GSSG/GSH ratio } \\
\text { as compared to non-frail }\end{array}$ \\
\hline & & & & & $\begin{array}{l}\text { Plasma MDA and HNE } \\
\text { proteins adducts }\end{array}$ & Spectrofluorimetry & $\begin{array}{l}\text { MDA and HNE adducts were } \\
\text { significantly higher in frailty as } \\
\text { compared to non-frail patients }\end{array}$ \\
\hline & & & & & $\begin{array}{l}\text { Plasma oxidized } \\
\text { proteins }\end{array}$ & Western Blot & $\begin{array}{l}\text { An appreciable decrease in } \\
\text { oxidized proteins was detected } \\
\text { in non-frail subjects when } \\
\text { compared to frail patients }\end{array}$ \\
\hline Wu et al. (2009) & $\mathrm{n}=90$ & Frail $n=21(79.9 \pm 5.8)$ & $\begin{array}{l}\text { Pre-frail } n=56 \\
(76.8 \pm 5.8) ; \\
\text { non-frail } n=13 \\
(73.1 \pm 5.3)\end{array}$ & Fried et al. (2001) & Serum 8-OHdG & ELISA & $\begin{array}{l}\text { Frail subjects had higher serum } \\
8 \text {-OHdGlevel than pre-frail and } \\
\text { non-frail individuals }\end{array}$ \\
\hline
\end{tabular}

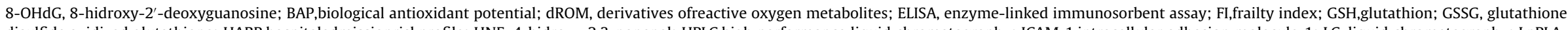

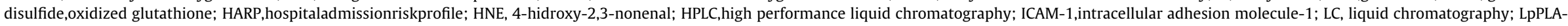

2,lipoprotein phospholipase A2; MCP-1,monocyte chemoattractant protein-1; MDA,malondialdehyde; MS, mass spectrometry; TBARS,thiobarbituricbindingacid reactivespecies; TTL,total thiol levels; Vit-E,vitamin E. 
Table 2

Studies assessing genomic biomarkers.

\begin{tabular}{|c|c|c|c|c|c|c|c|}
\hline Study & $\begin{array}{l}\text { Total population } \\
\text { (mean age } \pm \text { SD) }\end{array}$ & $\begin{array}{l}\text { Case population (mean } \\
\text { age } \pm \text { SD) }\end{array}$ & $\begin{array}{l}\text { Control population } \\
\text { (mean age } \pm \text { SD) }\end{array}$ & Frailty criteria & Outcomes & Assay method & Results \\
\hline Ashar et al. (2015) & $\begin{array}{l}\mathrm{n}=4892 ; 2774 \text { females, } \\
2118 \text { males }\end{array}$ & na & na & Fried et al. (2001) & mtDNA copy number & qPCR & $\begin{array}{l}\text { Lower mtDNA copy number } \\
\text { was significantly associated } \\
\text { with prevalent frailtyonly in } \\
\text { white participants }\end{array}$ \\
\hline \multirow[t]{2}{*}{ Bellizzi et al. (2012) } & $\begin{array}{l}\text { Cross-sectional study: } \\
\mathrm{n}=318 \text { ( } \mathrm{S} 1+\mathrm{S} 2 \text { ). } \\
\mathrm{S} 1: \mathrm{n}=217 \text { (median age } \\
75 \text { years) } 123 \text { females, } \\
94 \text { males, classified in } \\
\text { non-frail, pre-frail and } \\
\text { frail. } \\
\mathrm{S}: \mathrm{n}=101 \text { (median age } \\
99 \text { years) } 51 \text { females, } 50 \\
\text { males, classified in frail } \\
\text { and very frail }\end{array}$ & na & na & $\begin{array}{l}\text { Montesanto et al. } \\
\text { (2010) }\end{array}$ & $\begin{array}{l}\text { Global DNA } \\
\text { methylation }\end{array}$ & CpGlobal assay & $\begin{array}{l}\text { Global DNA methylation levels } \\
\text { were correlated with frailty in } \\
\text { S1 but not in S2. }\end{array}$ \\
\hline & $\begin{array}{l}\text { Longitudinal study } 7 \\
\text { years later: } \\
\mathrm{n}=37 \text {,random sample } \\
\text { from } S 1 \text { pre-frail and } \\
\text { non frail groups }\end{array}$ & & & & & & $\begin{array}{l}\text { Lower global DNA methylation } \\
\text { levels in pre-frail subjects that } \\
\text { became frail after } 7 \text { years }\end{array}$ \\
\hline Brault et al. (2014) & $\mathrm{n}=53(\geq 75$ years old $)$ & na & na & Fried et al. (2001) & $\begin{array}{l}\text { TL in leukocytes and } \\
\text { aortic tissue }\end{array}$ & & $\begin{array}{l}\text { No association between TL and } \\
\text { frailty. } \\
\text { Association between longer } \\
\text { leukocyte and aortic } \mathrm{T} / \mathrm{S} \text { ratio } \\
\text { and greater number of clinical } \\
\text { frailty criteria. }\end{array}$ \\
\hline \multirow[t]{2}{*}{ Breitling et al. (2016) } & $\begin{array}{l}\text { Dataset } 1: \mathrm{n}=969 \\
(62.1 \pm 6.5) 484 \\
\text { females, } 485 \text { males }\end{array}$ & na & na & FI (34 deficits) & $\begin{array}{l}\text { DNA methylation age } \\
\text { acceleration } \\
\text { (methylation age } \\
\text { minus chronological } \\
\text { age) }\end{array}$ & $\begin{array}{l}\text { Infinium } \\
\text { HumanMethylation } 450 \\
\text { BeadChip }\end{array}$ & $\begin{array}{l}\text { Association of DNA } \\
\text { methylation age acceleration } \\
\text { with FI increased with } \\
\text { increasing age acceleration }\end{array}$ \\
\hline & $\begin{array}{l}\text { Dataset } 2: \mathrm{n}=851 \\
(63 \pm 6.7) 464 \text { females, } \\
387 \text { males }\end{array}$ & & & & $\mathrm{TL}$ & qPCR & $\begin{array}{l}\text { Interaction between TL and } \\
\text { epigenetic age acceleration did } \\
\text { not improve the prediction of } \\
\text { FI }\end{array}$ \\
\hline
\end{tabular}


Table 2 (Continued)

\begin{tabular}{|c|c|c|c|c|c|c|c|}
\hline Study & $\begin{array}{l}\text { Total population } \\
\text { (mean age } \pm \mathrm{SD})\end{array}$ & $\begin{array}{l}\text { Case population (mean } \\
\text { age } \pm \text { SD) }\end{array}$ & $\begin{array}{l}\text { Control population } \\
\text { (mean age } \pm \text { SD) }\end{array}$ & Frailty criteria & Outcomes & Assay method & Results \\
\hline Collerton et al. (2012) & $\begin{array}{l}\mathrm{n}=552(85) 332 \\
\text { females, } 220 \text { males }\end{array}$ & $\begin{array}{l}\text { Frail } n=119(85) 92 \\
\text { females, } 27 \text { males }\end{array}$ & $\begin{array}{l}\text { Pre-frailn }=333(85) \\
193 \text { females, } 140 \\
\text { males; } \\
\text { non-frail } n=100(85) \\
47 \text { females, } 53 \text { males }\end{array}$ & $\begin{array}{l}\text { Fried et al. (2001) } \\
\text { FI ( } 40 \text { deficits) }\end{array}$ & $\begin{array}{l}\text { TL } \\
\text { Ionized } \\
\text { radiation-induced DNA } \\
\text { damage and repair }\end{array}$ & $\begin{array}{l}\text { qPCR } \\
\text { Automated } \\
\text { fluorimetric alkaline } \\
\text { DNA unwinding }\end{array}$ & $\begin{array}{l}\text { No association of TL or ionized } \\
\text { radiation-induced DNA } \\
\text { damage and repair with frailty }\end{array}$ \\
\hline Collerton et al. (2013) & $\mathrm{n}=1173(85.5)$ & $\begin{array}{l}\text { Frail } n=696 \text { (FI), } \\
n=477 \text { (Fried et al., } \\
\text { 2001) }\end{array}$ & $\begin{array}{l}3 \text { ethnically matched } \\
\text { population control data } \\
\text { sets }\end{array}$ & $\begin{array}{l}\text { Fried et al. (2001) } \\
\text { FI ( } 40 \text { deficits) }\end{array}$ & mtDNAhaplogroups & SequenomMassARRAY & $\begin{array}{l}\text { No association between } \\
\text { common genetic variants of } \\
\text { mtDNA and frailty }\end{array}$ \\
\hline Collerton et al. (2014) & $\begin{array}{l}\mathrm{n}=321(85) 184 \\
\text { females, } 137 \text { males } \\
\mathrm{n}=231(85) 148 \\
\text { females, } 83 \text { males }\end{array}$ & na & na & $\begin{array}{l}\text { Fried et al. (2001) (CHS } \\
\text { modified) }\end{array}$ & $\begin{array}{l}\text { DNA methylation in } \\
\text { CpG islands } \\
\text { LINE-1 methylation } \\
\text { levels (surrogate for } \\
\text { genome-wide DNA } \\
\text { methylation levels) }\end{array}$ & $\begin{array}{l}\text { Highly quantitative } \\
\text { pyrosequencing }\end{array}$ & $\begin{array}{l}\text { Association CpG island } \\
\text { methylation with frailty } \\
\text { No association genome-wide } \\
\text { methylation (LINE-1) with } \\
\text { frailty }\end{array}$ \\
\hline \multirow[t]{2}{*}{ Gao et al. (2017) } & $\begin{array}{l}\text { Discovery set } \mathrm{n}=978 \\
(62.1 \pm 6.5)\end{array}$ & Frail $n=100$ & $\begin{array}{l}\text { Pre-frail } n=443 \\
\text { non-frail } n=435\end{array}$ & FI (34 deficits) & $\begin{array}{l}\text { DNA methylation } \\
\text { profiles }\end{array}$ & $\begin{array}{l}\text { Illumina Human } \\
\text { Methylation } 450 \\
\text { BeadChip }\end{array}$ & $\begin{array}{l}17 \text { smoking-related CpG sites } \\
\text { were associated with the FI. } \\
9 \text { of those sites were }\end{array}$ \\
\hline & $\begin{array}{l}\text { Validation set } \mathrm{n}=531 \\
(62.0 \pm 6.6)\end{array}$ & Frail $n=48$ & $\begin{array}{l}\text { Pre-frail } n=220 \\
\text { non-frail } n=263\end{array}$ & & & & $\begin{array}{l}\text { designated as frailty associated } \\
\text { loci. Six of them [cg02657160 } \\
\text { (CPOX), cg05673882 (POLK), } \\
\text { cg07826859 (MYO1G), } \\
\text { cg19859270 (GPR15), } \\
\text { cg23667432 (ALPP), and } \\
\text { cg25189904 (GNG12)]were } \\
\text { mapped showing methylation } \\
\text { intensity in } \\
\text { frail < pre-frail < non-frail } \\
\text { SI, based on those 9 } \\
\text { smoking-related CpG sites, } \\
\text { manifested a monotonic } \\
\text { dose-response relationship } \\
\text { with the FI }\end{array}$ \\
\hline Ho et al. (2011) & $\mathrm{n}=348$ females $(74.2)$ & Frail $n=152$ & $\begin{array}{l}\text { Pre-frail } n=165 \\
\text { non-frail } n=32\end{array}$ & Fried et al. (2001) & $\begin{array}{l}\text { SNP variations (134 } \\
\text { genes, } 1354 \text { SNP) }\end{array}$ & $\begin{array}{l}\text { Bead Array (Illumina } \\
\text { custom GoldenGate } \\
1536 \text { SNP panel) }\end{array}$ & $\begin{array}{l}20 \text { SNP (from } 11 \text { genes) } \\
\text { associated with frailty (not } \\
\text { significantly) }\end{array}$ \\
\hline \multirow[t]{4}{*}{ Jylhava et al. (2013) } & $\mathrm{n}=174$ & $\begin{array}{l}\text { Frail } \mathrm{n}=144 \\
\text { (nonagerians) } 101 \\
\text { females, } 43 \text { males }\end{array}$ & $\begin{array}{l}\mathrm{n}=30 \text { young controls } \\
\text { (range } 19-30 \text { years) } 21 \\
\text { females, } 9 \text { males }\end{array}$ & Fried et al. (2001) & Total cf-DNA in plasma & $\begin{array}{l}\text { Fluorimetry } \\
\text { (Quant-iT }^{\mathrm{TM}} \text { DNA } \\
\text { high-sensitivity assay } \\
\text { kit) }\end{array}$ & $\begin{array}{l}\text { In nonagenarians: } \\
\text { Higher levels of total and } \\
\text { unmethylated cf-DNA were } \\
\text { associated with increased }\end{array}$ \\
\hline & & & & & Unmethylated cf-DNA & $\begin{array}{l}\text { ELISA (DNA } \\
\text { Methylation Kit) }\end{array}$ & frailty \\
\hline & & & & & $\begin{array}{l}\text { Genomic equivalents of } \\
\text { the RNase P-coding } \\
\text { cf-DNA }\end{array}$ & qPCR & No association with frailty \\
\hline & & & & & $\begin{array}{l}\text { Alu repeat cf-DNA } \\
\text { mtDNA copy number }\end{array}$ & $\begin{array}{l}\text { qPCR } \\
\text { qPCR }\end{array}$ & $\begin{array}{l}\text { No association with frailty } \\
\text { mtDNA copy number was } \\
\text { directly correlated with } \\
\text { increased frailty }\end{array}$ \\
\hline
\end{tabular}




\begin{tabular}{|c|c|c|c|c|c|c|c|}
\hline Kim et al. (2015) & $\begin{array}{l}\mathrm{n}=320 \text {. Parents at least } \\
90 \text { years old and their } \\
\text { offspring ( } 50-80 \text { years } \\
\text { old) }\end{array}$ & na & na & FI (34 deficits) & $\begin{array}{l}\text { Genome-wide linkage } \\
\text { scanning followed by } \\
\text { fine-scale association } \\
\text { mapping }\end{array}$ & $\begin{array}{l}\text { Genotyping: BeadChip } \\
\text { array (Illumina } \\
\text { Infinium Linkage } 24 \\
\text { set); } \\
\text { association mapping:I } \\
\text { lluminaGoldenGate } \\
\text { assay }\end{array}$ & $\begin{array}{l}\text { They found three sites } \\
\text { associated with healthy ageing }\end{array}$ \\
\hline Marzetti et al. (2014) & $\begin{array}{l}\mathrm{n}=142(74.9 \pm 6.5) 84 \\
\text { females, } 58 \text { males }\end{array}$ & Frail $n=74$ & $\begin{array}{l}\text { Pre-frail/non-frail: } \\
n=68\end{array}$ & $\begin{array}{l}\text { Fried et al. (2001) } \\
\text { FI (30 deficits) }\end{array}$ & $\mathrm{TL}$ & qPCR & $\begin{array}{l}\text { No association with frailty } \\
\text { (Fried or FI) }\end{array}$ \\
\hline Matteini et al. (2010) & $\mathrm{n}=326$ females $(74.1)$ & na & na & Fried et al. (2001) & $\begin{array}{l}56 \text { SNP from six } \\
\text { candidate genes } \\
\text { involved in Vitamin } \\
\text { B12metabolic } \\
\text { pathway: MTHFR, } \\
\text { MTR, MTRR, CBS, TCN1 } \\
\text { and TCN2 }\end{array}$ & Illumina BeadArray & $\begin{array}{l}\text { SNP in the TCN2 showed } \\
\text { significant association with } \\
\text { frailty. } \\
\text { Two SNP in MTRR showed 2-4 } \\
\text { times greater odds of being } \\
\text { frail compared to robust }\end{array}$ \\
\hline \multirow[t]{2}{*}{ Moore et al. (2010) } & Pilot study: $\mathrm{n}=315$ & $\begin{array}{l}\text { Frail } \mathrm{n}=154(75 \pm 4.45) \\
112 \text { females, } 42 \text { males. }\end{array}$ & $\begin{array}{l}\text { Non-frail: } \mathrm{n}=161 \\
(81.35 \pm 3.16) 111 \\
\text { females, } 50 \text { males }\end{array}$ & Fried et al. (2001) & $\begin{array}{l}\text { mtDNA variations } \\
\text { (SNP) }\end{array}$ & $\begin{array}{l}\text { Oligonucleotide } \\
\text { sequencing microarray }\end{array}$ & $\begin{array}{l}\text { Three mtDNA SNPs(mt } 146 \text {, } \\
\text { mt204, and mt } 228) \text { were } \\
\text { associated with frailty }\end{array}$ \\
\hline & $\begin{array}{l}\text { Cross-sectional study: } \\
\text { total } n=5275\end{array}$ & $\begin{array}{l}\text { Frail white: } \mathrm{n}=262 \\
(77.36 \pm 6.36) 174 \\
\text { females, } 88 \text { males; frail } \\
\text { black: } \mathrm{n}=102 \\
(75.44 \pm 6.76) 76 \\
\text { females, } 26 \text { males }\end{array}$ & $\begin{array}{l}\text { Non-frail white: } \\
\mathrm{n}=4223(72.43 \pm 5.37) \\
2377 \text { females, } 1846 \\
\text { males; non-frail black: } \\
\mathrm{n}=688(72.05 \pm 5.09) \\
425 \text { females, } 263 \text { males }\end{array}$ & & & $\begin{array}{l}\text { Real-time PCR TaqMan } \\
\text { assays for individual } \\
\text { SNP selected for } \\
\text { follow-up (mt146, } \\
\text { mt204, and mt228) }\end{array}$ & $\begin{array}{l}\text { mt204C allele was associated } \\
\text { with greater likelihood of } \\
\text { frailty }\end{array}$ \\
\hline \multirow[t]{2}{*}{ Saum et al. (2014) } & $\begin{array}{l}\mathrm{n}=3537(61.9 \pm 6.6) \\
1963 \text { females, } \\
\text { 1574males. }\end{array}$ & na & na & FI (34 deficits) & Relative $\mathrm{TL}$ ( $\mathrm{T} / \mathrm{S}$ ratio) & qPCR & $\begin{array}{l}\text { No difference of the FI between } \\
\text { the } \mathrm{T} / \mathrm{S} \text { ratio tertiles was } \\
\text { observed. }\end{array}$ \\
\hline & $\begin{array}{l}\text { Validation in a } \\
\text { subpopulation } \mathrm{n}=20\end{array}$ & & & & $\begin{array}{l}\text { Absolute TL in base } \\
\text { pairs }\end{array}$ & Southern blot & $\begin{array}{l}\text { TL measurements by the T/S } \\
\text { ratio were highly correlated } \\
\text { with absolute TL }\end{array}$ \\
\hline $\begin{array}{l}\text { Valdiglesias et al. } \\
\text { (2015) }\end{array}$ & $\mathrm{n}=180$ & $\begin{array}{l}\text { Frail } n=93 \\
(76.9 \pm 6.6) 52 \text { females, } \\
41 \text { males }\end{array}$ & $\begin{array}{l}\text { Non-frail } n=87 \\
(72.9 \pm 6.1) 50 \text { females, } \\
37 \text { males }\end{array}$ & Fried et al. (2001) & MN frequency & $\begin{array}{l}\text { CBMN with automated } \\
\text { scoring }\end{array}$ & $\begin{array}{l}\text { No association between MN } \\
\text { frequency and frailty }\end{array}$ \\
\hline Woo et al. (2008) & $\begin{array}{l}\mathrm{n}=2006.1030 \text { females } \\
(72.02 \pm 5.191), 976 \\
\text { males }(72.75 \pm 5.026)\end{array}$ & na & na & FI (17 deficits) & $\mathrm{TL}$ & qPCR & $\begin{array}{l}\text { No correlation between TL and } \\
\text { frailty }\end{array}$ \\
\hline Yu et al. (2015) & $\begin{array}{l}\mathrm{n}=2006(72.4 \pm 5.1) \\
1030 \text { females, } 976 \\
\text { males }\end{array}$ & Frailn $=127$ & $\begin{array}{l}\text { Pre-frail } \mathrm{n}=967 \\
\text { non-frail } \mathrm{n}=912 \text {, }\end{array}$ & Fried et al. (2001) & $\mathrm{TL}$ & qPCR & $\begin{array}{l}\text { No association between TL and } \\
\text { frailty at baseline, nor in } 4 \text { year } \\
\text { follow-up }\end{array}$ \\
\hline
\end{tabular}

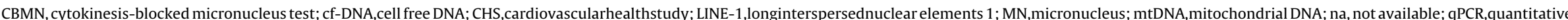
PCR; SI, Smoking index; SNP,singlenucleotidepolymorphism; T/S ratio,mean telomere repeat copy to single gene copy number; TL,telomere length. 
but their production can also be induced by exogenous agents that include ionizing radiation, air pollution and a wide range of chemicals (Halliwell, 2007). In such cases, the imbalance between increased ROS production and antioxidant defenses leads to an oxidative stress state which is highly destructive for the cell and the organism. It has been demonstrated that oxidative stress plays an important role in neurodegenerative diseases, often associated with ageing (Migliore and Coppede 2002; Perry et al., 2002); indeed oxidative DNA damage is one of the events that can be detected earlier in the pathogenesis of these diseases. Furthermore, oxidative stress is considered a risk factor for ageing (Coppedè and Migliore, 2009).

Besides, increases in oxidative stress with ageing may also contribute to the development of chronic inflammation and disease (Woods et al., 2012). Indeed, there are a variety of potential mechanisms linking oxidative stress to inflammation, including disturbances in the redox equilibrium, decrease of oxidationsensitive biomolecules related to immune response, such as vitamins or 5,6,7,8-tetrahydrobiopterin (BH4), or alterations in pattern recognition receptors of the innate immune system, such as toll-like receptors (Gill et al., 2010; Strasser et al., 2017). Accordingly, a number of previous studies have related immune system alterations to frailty (reviewed in Fulop et al., 2015). Still, this kind of immunological alterations, although may be classified as cellular alterations in some cases, were considered biomarkers at systematic level and, consequently, not included in the present review.

The relationship between oxidative stress and frailty has been studied in several works. Table 1 shows all human population studies evaluating the possible association between markers of oxidative stress and frailty in old age. A total of 9 studies were found in the literature addressing this issue. The total antioxidant cellular ability or the levels of some specific antioxidants in the frail subjects regarding the pre-frail and non-frail individuals was measured in 4 (44.4\%) out of 9 reviewed studies. In 7 studies, direct effects of ROS on lipids (71\%) (Collerton et al., 2012; Ingles et al., 2014; Liu et al., 2016; Pereira et al., 2016; Serviddio et al., 2009), proteins (57\%) (Inglés et al., 2014; Pereira et al., 2016; Serviddio et al., 2009) and DNA (29\%) (Namioka et al., 2016; Wu et al., 2009) were evaluated; evaluation of more than one target in the same study was common. Moreover, just in 2 cases (Namioka et al., 2016; Saum et al., 2015), the levels of derivatives of reactive oxygen metabolites (d-ROM) were determined.

Results from these studies do not clarify if oxidative stress is consequence of frailty (or vice versa), or if - as suggested by Liu et al. (2016) - there is a bidirectional relationship where the presence of one of them increases the risk of the other.

Cells have different mechanisms to limit the levels of ROS and the damage they induce. The cellular defense mechanisms against oxidative stress include both enzymatic (e.g. catalase, superoxide dismutase) and non-enzymatic (e.g. vitamin E [vit-E], glutathione) antioxidants, which play a central role in maintaining the cellular redox balance essential for cell survival (Birben et al., 2012). Several alterations in different antioxidants in association with frailty have been obtained in the studies reviewed. For instance, vit-E, or $\alpha$-tocopherol, is the major lipophilic antioxidant in humans; low levels of vit-E are considered an indirect biomarker of oxidative stress and, therefore, it has been hypothesized to be associated with an increased risk of frailty. According to this, Ble et al. (2006) found a significant decrease of vit-E levels in frail individuals, after adjustment for multiple confounders, in a population of 827 older individuals classified according to Fried's criteria.

Also, increases of both oxidized glutathione (GSSG) and the oxidized/reduced glutathione ratio (GSSG/GSH), but normal reduced glutathione (GSH) levels, were found in 43 frail individuals (Fried's criteria) regarding 19 non-frail subjects (Serviddio et al., 2009).
Investigating the association between oxidative stress and frailty, Saum et al. (2015) evaluated three biomarkers of oxidative stress in 2518 individuals classified according to Fried's criteria: total thiol levels (TTL), d-ROM, and biological antioxidant potential (BAP). Whilst no statistically significant difference was observed in the levels of BAP in frail individuals as compared with the prefrail and non-frail subjects, the frail group showed higher d-ROM but lower TLL levels. Also, significant positive correlations between $\mathrm{d}-\mathrm{ROM}$ and BAP, and BAP and TTL were observed, as well as a weak inverse association between d-ROM and TTL. The authors concluded that TTL was the biomarker most consistently associated with frailty in all the regression models.

Namioka et al. (2016) studied oxidative stress in a sample of 140 older adults with mild-to-moderate Alzheimer Disease, classified according to their frailty status using the Fried's criteria. To that end, levels of several oxidative stress biomarkers were evaluated including d-ROM, BAP, and endogenous plasma antioxidants (namely albumin, bilirubin, and uric acid). Consistently with the observations by Saum et al. (2015), results showed significantly increasing levels of dROM in non-frail < pre-frail < frail groups. On the other hand, BAP and plasma bilirubin levels were significantly lower in the frail group. Plasma albumin and uric acid levels showed no significant differences among the three groups.

ROS attack all biological molecules including DNA, proteins and lipids. Lipids and lipoproteins are particularly susceptible to ROS attack because hydrogen abstraction by a radical can initiate a devastating chain reaction: lipid peroxidation (Anderson and Phillips, 1999). Several studies evaluated lipid peroxidation and oxidized protein levels in relation to frailty. Serviddio et al. (2009) analyzed plasma levels of malonaldehyde (MDA) and 4-hydroxy2,3-nonenal (HNE) protein adducts (both of them recognized biomarkers of lipid peroxidation), and plasma oxidized proteins (as expression of oxidative protein damage) in a population of 62 old individuals, classified as frail and non-frail accordingly to the Fried's criteria. Higher levels of MDA and HNE adducts and oxidized proteins were observed in frail individuals as compared with non-frail subjects.

Liu et al. (2016) studied the association of frailty (Fried's criteria) with several oxidative stress biomarkers related to cardiovascular disease, namely isoprostanes (accurate markers of lipid peroxidation) and the lipoprotein phospholipase A2 (LpPLA2) (an enzyme involved in hydrolization of oxidized phospholipids) in a population of 1919 older individuals. They concluded that significantly increased levels of isoprostanes and LpPLA2 mass were related to greater odds of frailty, since they found individual association of frailty with elevated levels of isoprostanes and LPPLA2 mass, and of pre-frailty with elevated levels of LPPLA2 activity.

Inglés et al. (2009) employed MDA and protein carbonylation (a circulating indicator of oxidative damage to proteins) to assess the relationship between frailty and oxidative stress, and the capacity of these two parameters as possible biomarkers of frailty. Their results showed significantly higher levels of MDA and carbonylated proteins in older individuals classified as frail, according to Fried's criteria, than in non-frail; no relationship with age or sex was found in any case.

Also, Pereira et al. (2016) found in a small population $(n=15)$ that older individuals with a higher degree of frailty, measured by FI or HARP criteria, showed higher levels of lipid peroxidation. In this study, protein oxidation levels (MDA protein adducts) were also evaluated, showing higher levels in individuals with higher risk of frailty determined by HARP criteria, but not by FI.

Collerton et al. (2012) carried out a study with 552 older adults classified by means of both Fried's criteria and FI (40 items), evaluating the possible association between frailty status and biomarkers of lipid peroxidation, namely isoprostanes iPF2 
alpha-III and iPF2 alpha-VI. No association between frailty and the oxidative stress biomarkers was found in this case.

As previously indicated, ROS may react with different biomolecules in cells, and one of the main targets is DNA. This kind of damage is often referred to as oxidative DNA damage. The consequences include different types of DNA alterations, ranging from simple oxidation of bases to large deletions, through single and double strand breaks (Rao, 2009). Pathogenic roles for DNA oxidation include the induction of mitochondrial dysfunction, promotion of cytotoxicity and modulation of inflammatory responses (Evans and Cooke, 2006). According to our revision, onlytwo studies have evaluated consequences of oxidative stress on DNA in frail and non-frail older people. Wu et al. (2009) evaluated the relationship between oxidative stress and frailty by measuring the level of serum 8-hidroxy-2'-deoxyguanosine (8-OHdG) in a sample of Chinese older individuals $(n=90)$. The frailty status was determined by using the Fried's criteria. Results showed significantly increased serum 8-OHdG levels in frail individuals with respect to the prefrail and non-frail subjects. More recently, Namioka et al. (2016) observed that urinary excretions of 8 -OHdG and 8-isoprostane were significantly higher in frail and pre-frail individuals than those determined in the non-frail group.

Together with these 9 selected studies, two additional epidemiological studies, not included in the final reviewed works because they did not strictly fulfill the inclusion criteria, addressed the relationship between frailty and oxidative stress in an indirect way. Firstly, Caballero et al. (2014) assessed the role of oxidative stress on deficiencies of functional physical performance of the lower and/or upper body limbs, that could affect a future pre-frailty phenotype (Fried's criteria). To this aim, they studied the concentrations of plasma proteins, carbonylated proteins, lipid peroxidation and plasma total antioxidant activity, as measures of oxidative damage, in a population of 200 individuals aged $\geq 70$ years. Their results showed significantly lower levels of total antioxidant capacity in women $\leq 76$ years old with deficiencies in the physical performance of both lower and upper body limbs, but not in women $>76$ years old or in men of any age, suggesting that deficient oxidative defense in the elderly could significantly affect the functional physical performance and future outcomes of pre-frail individuals.

Secondly, Baptista et al. (2012) used the gait speed as a measure of physical performance in older adults, in a population of 280 individuals. The objective of this study was to evaluate the superoxide anion production, and its interaction with physical frailty measured by gait speed in older adults. They found no differences in the baseline levels of superoxide anion production between subjects with slower and faster gait speed. However, after stimulation with PMA (lucigenin and phorbol 12-myristate 13-acetate), the superoxide anion production was significantly higher in slow walkers (gait speed $<0.8 \mathrm{~m} / \mathrm{s}$ ). Hence, they suggested that the production of the superoxide anion can be involved in the decline of physical performance in the older age and, therefore, in the frailty process.

\subsection{Genomic biomarkers}

The contribution of the individual genetic profile to the development of frail is still uncertain. Whether and to what extent the individual genetic features affect the individual susceptibility to frailty is not well established yet. In this regard, studies involving analysis of the contribution of genetic background to frailty and in particular evaluating the association of genomic instability to this condition may help to provide insights into biologically relevant pathways that contribute to frailty. Among the studies reviewed here addressing genetic alterations, six of them (35.3\%) evaluated genetic background of frail individuals, including variations in both nuclear and mitochondrial DNA, eight (47\%) investigated the relationship between genomic instability and frailty, and three $(17,7 \%)$ addressed the possible epigenetic characteristics of frailty status (Table 2).

\subsubsection{Individual genetic background}

Three out of the 17 studies reviewed here evaluated the individual genetic differences associated to frailty. Kim et al. (2015) studied the heritability of healthy ageing as an attempt to find the more beneficial genetic variants or less disadvantageous variants present in healthy, long-lived people. The authors performed a genome-wide linkage analysis and fine-scaled association mapping of linkage regions, using the FI (34 deficits) as a quantitative measure of frailty in a population of 320 older adults. Three healthy ageing sites (HAS) were found at 12q13-14 in intergenic regions (HAS- 1 and -2 with enhancer activity, HAS- 3 with silencer activity).

Ho et al. (2011) studied different biological pathways involved in frailty to try to find variations in genes related to the frailty syndrome. To this aim, they genotyped 1354 SNP from 134 candidate genes involved in inflammation and muscle maintenance, two of the main phenotypic characteristics of frailty, in a population of 348 subjects classified according to Fried's criteria. Twenty SNP, indirectly related to inflammatory process, were found to be associated with frailty, although statistical significance was not reached.

Matteini et al. (2010) also investigated genetic variants of six candidate genes (MTHFR, MTR, MTRR, CBS, TCN1, and TCN2) involved in Vitamin B12 metabolic pathway and their association with frailty (Fried's criteria), in 416 older women. For this purpose 56 SNP from those six candidate genes were genotyped. The results found SNP in the TCN2 gene showing significant association with frailty. Moreover, two SNP in MTRR gene showed 2-4 times greater odds of being frail compared to robust.

Mitochondria are involved in several major cell functions, such as cell production of energy, metabolic and apoptotic processes, as well as being a major site of ROS generation. Mitochondrial function experiences alterations with age and these changes are associated with several age-related diseases. Thus, age-related changes and variations in the mtDNA have been proposed as plausible candidates to play a role in degenerative and senescent processes, and to contribute to increases in vulnerability in late life (Moore et al., 2010); hence they are likely to be associated with frailty syndrome. Three studies were found in the literature search that evaluated the possible association between mtDNA alterations (in copy number or in sequence) and frailty. Firstly, Collerton et al. (2013) evaluated the association between common genetic variants of mtDNA and frailty as a mean to study heritability of human longevity and healthy ageing, since frailty is considered an "unhealthy ageing" phenotype. They evaluated a population of 1173 older adults. No association between mtDNA haplogroups and frailty, assessed by both Fried's criteria and FI (40 deficits), was observed.

On the contrary, Ashar et al. (2015) evaluated the association between mtDNA copy number and prevalent frailty (Fried's criteria; $n=4892$ ), reporting lower mtDNA copy number in frail white, but not in frail black, individuals. They also observed a significant inverse association of mtDNA copy number with age, and higher mtDNA copy number in women relative to men. Finally, Moore et al. (2010) studied how mtDNA variations (SNP) with age may increase the susceptibility to frailty. In a pilot study of 315 individuals classified according to Fried's criteria, three mtDNA SNP were associated with frailty (mt146, mt204, and mt228). From these three SNP, $\mathrm{mt} 204 \mathrm{C}$ was confirmed to be the allele associated with greater likelihood of frailty in the cross-sectional further study including 5275 subjects.

After cell damage or death, DNA is released into the circulation; this is why plasma cell free DNA (cf-DNA) reflects systematic inflammation and cell death, what makes it a potential biomarker of ageing and frailty. For that reason, Jylhava et al. (2013) quantified 
the plasma levels of total cf-DNA, unmethylated cf-DNA, genecoding cf-DNA, andAlu repeat cf-DNA, and mtDNA copy number to study their potential as biomarkers of frailty (Fried's criteria; $n=174$ ). Their results showed increased levels of total and unmethylated cf-DNA associated with frailty. Also mtDNA copy number was correlated with frailty. However, neither gene-coding cf-DNA nor Alu repeat cf-DNA were associatedwith frailty. Similarly, the same group (Jylhava et al., 2014) studied the association of these biomarkers with mortality, finding that frailty is detrimental for survival when used to adjust the results obtained.

\subsubsection{Genomic instability}

As mentioned before, eight out of 17 studies evaluated genomic instability in frailty. Genomic instability refers to a set of genetic events capable of causing temporary or permanent unscheduled alterations within the genome, including diverse types of chromosomal alterations (e.g. inversions, deletions, duplications and translocations of large chromosomal segments) (Migliore et al., 2011; Valdiglesias et al., 2015). Thus, genome instability could lead to altered gene dosage and gene expression as well as contribute to the risk of accelerated cell death in neuronal tissue (Thomas and Fenech, 2007). In accordance, genomic instability is considered a hallmark of a number of ageing-related diseases including cancer, and is related to the ageing phenotype and neurodegenerative disorders (Coppedè and Migliore, 2010; Migliore et al., 2011),

Among the studies evaluating genomic instability, all but one (Valdiglesias et al., 2015) analyzed telomere length (TL) in the older subjects. TL analysis is a common approach to evaluate genomic instability in blood samples. Telomeres are regions of repetitive nucleotide sequence at each end of the chromosomes, which contribute to maintain their integrity. They progressively shorten as the cell divides, limiting the number of divisions that normal somatic cells can undergo (Marzetti et al., 2014). The observation that telomeres shorten over the life course has led to the hypothesis that telomere attrition may be a mechanism driving the ageing process (Mikhelson and Gamaley, 2012). Nevertheless, all the studies reviewed here failed in finding an association between frailty and TL shortening: Collerton et al. (2012), using both Fried's criteria and FI (40 deficits; $n=552)$, Marzetti et al. (2014) ( $n=142)$ and Yu et al. (2015) ( $\mathrm{n}=2006)$ employing the Fried's criteria, Saum et al. (2014) $(\mathrm{n}=3537)$ and Woo et al. (2008) $(\mathrm{n}=2006)$ using the FI (34 and 17 deficits, respectively). Lack of association between TL and frailty (Fried's criteria) was also obtained by Brault et al. (2014) in older subjects with cardiovascular disease $(n=53)$; however, in this case, they found an unexpected association between longer leukocyte and aortic $\mathrm{T} / \mathrm{S}$ ratio(mean telomere repeat copy to single gene copy number) and greater number of clinical frailty criteria. Together with these studies, Breitling et al. (2016) evaluated the possible association between TL and DNA methylation age acceleration (methylation age minus chronological age) in a population of 851 older adults. They found no correlation between the two parameters, supporting the previously mentioned observations and the idea that TL is not a good biomarker for the identification of frailty.

The frequency of micronucleus (MN) in peripheral blood lymphocytes is also a biomarker of genomic stability widely employed in molecular epidemiology. Similarly to TL, MN frequency has been previously associated with age-related diseases and the process of ageing (Bonassi et al., 2001; Migliore et al., 2011). Valdiglesias et al. (2015) evaluated the frequency of MN to study the association of genomic instability and frailty in a population of 180 older adults classified according to Fried's criteria. Again, no association between MN frequency and frailty was observed in this case.

Thus, despite the well-reasoned working hypothesis (genomic instability is involved in the pathogenesis of frailty syndrome), all these studies resulted surprisingly fruitless, with no positive association between genomic instability and frailty severity. Two possible explanations were suggested for this lack of association (Valdiglesias et al., 2015). Firstly, the negative findings reported in all these studies may be caused by the high rate of basal genomic damage present in healthy older individuals and reported in previous studies (Mladinic et al., 2010). And secondly, this lack of association may be due to the physiological accumulation of genome damage in the elderly, which could limit the rate of genomic damage production. This condition could, for instance, limit the rate of MN formation.

\subsubsection{Epigenetics}

It has been previously reported that the genetic material of cells experiences epigenetic variations during the ageing process (reviewed in Sen et al., 2016). A total of 3 studies out of 17 evaluating genomic biomarkers, addressed the study of epigenetics in frail older adults. The three of them showed positive correlation between frailty status and DNA methylation. Bellizzi et al. (2012) reported the first study investigating the possible correlation between age-related functional decline, including frailty status, and epigenetic modifications. They measured global DNA methylation levels in a sample of 318 older people, divided in middle/advanced-aged subjects (median age 75 years) and ultranonagenarians (median age 99 years) in a cross-sectional study, and in a 7 year follow-up of a subsample of pre-frail and nonfrail middle aged subjects. Frailty status was determined using the Montesanto et al. (2010) scale, a hierarchical cluster analysis. Higher values of global DNA methylation were observed in frail middle aged subjects respect to pre-frail and non-frail, but no difference between very frail and frail was observed in the ultranonagenarians. In the follow-up study, those individuals that became frail after 7 years showed a significant increase of DNA methylation levels.

More recently, Breitling et al. (2016) evaluated the association between DNA methylation age acceleration and frailty in a population of 969 older adults, classified according their frailty status by using the FI (34 deficits). Their findings showed significantly increasing accumulation of frailty deficits with increasing methylation age acceleration, supporting an association between epigenetic age acceleration and frailty status. Also on this regard, Gao et al. (2017) examined the associations of smoking-related DNA methylation biomarkers and frailty in a population of older adults classified according to FI, and observed that methylation intensity of each locus in the validation panel was significantly lower in the frail, when compared to non-frail, population, whereas intermediate levels of methylation intensity were observed in the pre-frail subjects. On the basis of their results, authors suggested that $\mathrm{CpG}$ sites identified could have the potential to be prognostic biomarkers of frailty or frailty-related health outcomes.

Following the same assumption that DNA methylation changes with age, especially in gene promoter regions, Collerton et al. (2014) studied the importance of altered DNA methylation in frailty in 552 subjects (Fried's criteria). To this end, methylation at specific cytosine residues within CpG islands associated with gene transcriptional start sites was quantified. Furthermore, to estimate the genome-wide DNA methylation levels, they quantified methylation at LINE-1 repetitive elements as a surrogate, showing no association with frailty. However, a clear association between CpG island methylation and frailty was observed, suggesting a potential role for age-related ranges in CpG island methylation in the development of frailty.

\subsection{DNA repair ability}

The DNA repair system has been recognized as one of the most important cellular defense mechanisms responsible for the integrity of DNA. Decreased DNA repair ability is exhibited in vari- 
ous clinical conditions and associated with increased frequency of carcinogenesis, since inefficient repair leads to an accumulation of aberrations in the genome that culminate in the genetic instability typical of many malignancies and other pathologies (Valdiglesias et al., 2011).

Daily exposure to environmental agents (such as oxidizing chemicals, methylating agents, UV light, and ionizing radiation), and even normal physiological processes (such as replication and recombination), all may damage cellular components, including DNA. While modified proteins and lipids can be degraded and resynthesized, DNA must be repaired before replication and cell division take place (Klungland and Bjelland, 2007). Toxic and mutagenic consequences are minimized by distinct pathways of cellular repair that include different enzymes and protein complexes encoded by a number of human DNA repair genes. Genomic instability, previously described, is highly related to failures in cellular repair since aberrant DNA polymerases and other components of the transcriptional and translational machinery are accumulated with age (Rattan, 2012). Thus, during ageing, accumulation of mistakes takes place in the genetic material due to the loss of efficiency of these DNA repair systems, and contributes to the development of genomic instability. Indeed, most age-related diseases and ageing signs are associated with genomic instability and with unrepaired or erroneously repaired genome damage (Bürkle, 2001). Besides, it is known that the accumulation of DNA damage is involved in premature ageing and neurodegenerative processes, suggesting that alterations in the DNA repair mechanisms may be relevant to these disorders (Coppedè and Migliore, 2010). Indeed, the impact of cellular senescence on ageing of organisms was previously evaluated in different studies revealing an accumulation of DNA damage in both senescent cells and ageing organisms (Sedelnikova et al., 2008). In particular, several works described accumulated $\gamma \mathrm{H} 2 \mathrm{AX}$ foci, which reveal persistent DNA double-stranded breaks, in senescing human cell cultures and in ageing mice (Sedelnikova et al., 2004), in early thymocyte subsets of aged as compared to young mice (Hesse et al., 2009), in different organs from ageing C57Bl6 mice (Wang et al., 2009) and in fibroblasts taken from patients with Werner syndrome (Sedelnikova et al., 2008).

To our knowledge, the only study that evaluates DNA damage and repair capacity in association with frailty in human populationbased studies is the one reported by Collerton et al. (2012), where no association was observed using both Fried's criteria and FI (40 deficits) to identify frailty status.

\section{Conclusions}

Frailty is gaining attention in the last decades in geriatrics and research areas, with more and more professionals claiming frailty measurement to be incorporated into clinical practice as part of routine care for older patients. However, the currently used criteria identify frailty only after clinical manifestations are obvious. Increasing evidence suggest that the clinical concept of frailty -based mainly on phenotypical signs and symptoms and barely considering its biological basis - is obsolete since no single altered system alone defines frailty, but multiple systems are involved in this syndrome.

In order to achieve a more thorough and objective assessment for early identification of frailty, it is necessary to develop new tools that allow to recognize those individuals more vulnerable and more prone to develop the frailty syndrome. Within this framework, cellular and molecular biomarkers could be used to reach a more accurate identification of frailty, as well as those individuals in early and potentially reversible frailty stages (pre-frail individuals). The development of these new tools and their inclusion in the criteria to identify frailty would facilitate the implementation of personalized care and treatments, as well as improve outcomes by means of prevention and intervention programmes. Additional research is needed to further explore the pathophysiological bases of frailty.

In this review, population studies evaluating alterations associated with frailty status at cellular and molecular level - by means of oxidative stress, genomic and DNA repair biomarkers - were revised and compared in order to gather all this information and to identify potential biomarkers that could be useful in frailty identification, as well as to point out gaps of knowledge and new research areas needed in this field. Results of this revision showed that several oxidative stress biomarkers -including alterations in antioxidant systems, increased levels of lipid peroxidation and DNA oxidative damage, as well as DNA methylation and some specific genetic polymorphisms -are associated with frailty status in older people. On the contrary genomic instability, or at least the two biomarkers tested so far (telomere length and MN rate) seems not to be linked to frailty. The only study which addressed the possible relationship between DNA repair modulations and frailty status also failed in finding associations.

Despite the number of cellular alterations initially associated with frailty, studies on this regard are still very scarce and limited to some of the possible cellular targets. Additional research is needed to further explore these alterations prior to include any of them in the frailty assessment criteria. However, given the solid link between DNA repair ability, genomic instability, and age and age-related disorders, deeper investigations in this line must be carried out before reaching solid conclusions.

\section{Acknowledgments}

Research funded by Xunta de Galicia (ED431B 2016/013) and by EU COST Action IS1402. V. Valdiglesias was supported by a Xunta de Galicia postdoctoral fellowship (reference ED481B 2016/190-0). D. Marcos-Pérez and M. Sánchez-Flores were supported by INDITEXUDC fellowships. The work of S. Bonassi was supported by grants funded by the Associazione Italiana per la Ricerca sul Cancro (AIRC).

\section{References}

Abellan van Kan, G., Rolland, Y.M., Morley, J.E., Vellas, B., 2008. Frailty: toward a clinical definition. J. Am. Med. Dir. Assoc. 9, 71-72.

Anderson, D., Phillips, B., 1999. Comparative in vitro and in vivo effects of antioxidants. Food Chem. Toxicol. 37, 1015-1025.

Ashar, F.N., Moes, A., Moore, A.Z., Grove, M.L., Chaves, P.H., Coresh, J., Newman, A.B., Matteini, A.M., Bandeen-Roche, K., Boerwinkle, E., Walston, J.D., Arking, D.E., 2015. Association of mitochondrial DNA levels with frailty and all-cause mortality. J. Mol. Med. 93, 177-186.

Baptista, G., Dupuy, A.M., Jaussent, A., Durant, R., Ventura, E., Sauguet, P., Picot, M.C., Jeandel, C., Cristol, J.P., 2012. Low-grade chronic inflammation and superoxide anion production by NADPH oxidase are the main determinants of physical frailty in older adults. Free Radic. Res. 46, 1108-1114.

Bellizzi, D., D’Aquila, P., Montesanto, A., Corsonello, A., Mari, V., Mazzei, B., Lattanzio, F., Passarino, G., 2012. Global DNA methylation in old subjects is correlated with frailty. Age (Dordr) 34, 169-179.

Birben, E., Sahiner, U.M., Sackesen, C., Erzurum, S., Kalayci, O., 2012. Oxidative stress and antioxidant defense. World Allergy Organ. J. 5, 9-19.

Ble, A., Cherubini, A., Volpato, S., Bartali, B., Walston, J.D., Windham, B.G., Bandinelli, S., Lauretani, F., Guralnik, J.M., Ferrucci, L., 2006. Lower plasma vitamin $\mathrm{E}$ levels are associated with the frailty syndrome: the InCHIANTI study. J. Gerontol. A Biol. Sci. Med. Sci. 61, 278-283.

Bonassi, S., Fenech, M., Lando, C., Lin, Y.P., Ceppi, M., Chang, W.P., Holland, N., Kirsch-Volders, M., Zeiger, E., Ban, S., Barale, R., Bigatti, M.P., Bolognesi, C., Jia, C., Di Giorgio, M., Ferguson, L.R., Fucic, A., Lima, O.G., Hrelia, P., Krishnaja, A.P. Lee, T.K., Migliore, L., Mikhalevich, L., Mirkova, E., Mosesso, P., Muller, W.U. Odagiri, Y., Scarffi, M.R., Szabova, E., Vorobtsova, I., Vral, A., Zijno, A., 2001. HUmanMicroNucleus project: international database comparison for results with the cytokinesis-block micronucleus assay in human lymphocytes: I Effect of laboratory protocol, scoring criteria, and host factors on the frequency of micronuclei. Environ. Mol. Mutagen. 37, 31-45.

Brault, M.E., Ohayon, S.M., Kwan, R., Bergman, H., Eisenberg, M.J., Boivin, J.F., Morin, J.F., Langlois, Y., Autexier, C., Afilalo, J., 2014. Telomere length and the clinical phenotype of frailty in older adults undergoing cardiac surgery. J. Am. Geriatr. Soc. $62,2205-2207$ 
Breitling, L.P., Saum, K.U., Perna, L., Schottker, B., Holleczek, B., Brenner, H., 2016. Frailty is associated with the epigenetic clock but not with telomere length in a German cohort. Clin. Epigenetics 8, 21

Caballero, B., Rubio-Gonzalez, A., Potes, Y., Martinez-Reig, M., Sanchez-Jurado, P.M., Romero, L., Solano, J.J., Abizanda, P., Coto-Montes, A., 2014. Associations of the antioxidant capacity and hemoglobin levels with functional physical performance of the upper and lower body limbs. Age (Dordr) 36, 851-867.

Clegg, A., Young, J., Iliffe, S., Rikkert, M.O., Rockwood, K., 2013. Frailty in elderly people. Lancet 381, 752-762

Collerton, J., Martin-Ruiz, C., Davies, K., Hilkens, C.M., Isaacs, J., Kolenda, C., Parker C., Dunn, M., Catt, M., Jagger, C., von Zglinicki, T., Kirkwood, T.B., 2012. Frailty and the role of inflammation, immunosenescence and cellular ageing in the very old: cross-sectional findings from the Newcastle 85+ Study. Mech. Ageing Dev. 133, 456-466.

Collerton, J., Ashok, D., Martin-Ruiz, C., Pyle, A., Hudson, G., Yadegarfar, M., Davies, K., Jagger, C., von Zglinicki, T., Kirkwood, T.B., Chinnery, P.F., 2013. Frailty and mortality are not influenced by mitochondrial DNA haplotypes in the very old. Neurobiol. Aging 34 (288), e2881-2884.

Collerton, J., Gautrey, H.E., van Otterdijk, S.D., Davies, K., Martin-Ruiz, C., von Zglinicki, T., Kirkwood, T.B., Jagger, C., Mathers, J.C., Strathdee, G., 2014. Acquisition of aberrant DNA methylation is associated with frailty in the very old: findings from the Newcastle 85+ Study. Biogerontology 15, 317-328.

Coppedè, F., Migliore, L., 2009. DNA damage and repair in Alzheimer's disease. Curr. Alzheimer Res. 6, 36-47.

Coppedè, F., Migliore, L., 2010. DNA repair in premature aging disorders and neurodegeneration. Curr. Aging Sci. 3, 3-19.

Dent, E., Kowal, P., Hoogendijk, E.O., 2016. Frailty measurement in research and clinical practice: a review. Eur. J. Intern. Med. 31, 3-10.

Dulac, M.C., Aubertin-Leheudre, M., 2016. Exercise: an important key to prevent physical and cognitive frailty. J. Frailty Aging 5, 3-5.

Espinoza, S.E., Jung, I., Hazuda, H., 2012. Frailty transitions in the San Antonio Longitudinal Study of Aging. J. Am. Geriatr. Soc. 4, 652-660.

Evans, M.D., Cooke, M.S., 2006. Oxidative damage to DNA in non-malignant disease: biomarker or biohazard? Genome Dyn. 1, 53-66.

Fielding, R.A., 2015. A summary of the biological basis of frailty. Nestle Nutr. Inst. Workshop Ser. 83, 41-44.

Fried, L.P., Tangen, C.M., Walston, J., Newman, A.B., Hirsch, C., Gottdiener, J., Seeman, T., Tracy, R., Kop, W.J., Burke, G., McBurnie, M.A., 2001. Frailty in older adults: evidence for a phenotype. J. Gerontol. A Biol. Sci. Med. Sci. 56, M146-156.

Fried, L.P., Xue, Q.L., Cappola, A.R., Ferrucci, L., Chaves, P., Varadhan, R., Guralnik, J.M., Leng, S.X., Semba, R.D., Walston, J.D., Blaum, C.S., Bandeen-Roche, K., 2009. Nonlinear multisystem physiological dysregulation associated with frailty in older women: implications for etiology and treatment. J. Gerontol. A Biol. Sci. Med. Sci. 64, 1049-1057

Fulop, T., McElhaney, J., Pawelec, G., Cohen, A.A., Morais, J.A., Dupuis, G., Baehl, S. Camous, X., Witkowski, J.M., Larbi, A., 2015. Frailty, inflammation and immunosenescence. Interdiscip. Top Gerontol. Geriatr. 41, 26-40.

Gao, X., Zhang, Y., Saum, K.U., Schottker, B., Breitling, L.P., Brenner, H., 2017. Tobacco smoking and smoking-related DNA methylation are associated with the development of frailty among older adults. Epigenetics 12, 149-156.

Gill, T.M., Gahbauer, E.A., Allore, H.G., Han, L., 2006. Transitions between frailty states among community-living older persons. Arch. Intern. Med. 166, 418-423.

Gill, R., Tsung, A., Billiar, T., 2010. Linking oxidative stress to inflammation: toll-like receptors. Free Radic. Biol. Med. 48, 1121-1132.

Halliwell, B., 2007. Biochemistry of oxidative stress. Biochem. Soc. Trans. 35, $1147-1150$

Hesse, J.E., Faulkner, M.F., Durdik, J.M., 2009. Increase in double-stranded DNA break-related foci in early-stage thymocytes of aged mice. Exp. Geront. 44, 676-684.

Ho, Y.Y., Matteini, A.M., Beamer, B., Fried, L., Xue, Q.L., Arking, D.E., Chakravarti, A., Fallin, M.D., Walston, J., 2011. Exploring biologically relevant pathways in frailty. J. Gerontol. A Biol. Sci. Med. Sci. 66, 975-979.

Ingles, M., Gambini, J., Carnicero, J.A., Garcia-Garcia, F.J., Rodriguez-Manas, L., Olaso-Gonzalez, G., Dromant, M., Borras, C., Vina, J., 2014. Oxidative stress is related to frailty, not to age or sex, in a geriatric population: lipid and protein oxidation as biomarkers of frailty. J. Am. Geriatr. Soc. 62, 1324-1328.

Jylhava, J., Nevalainen, T., Marttila, S., Jylha, M., Hervonen, A., Hurme, M., 2013. Characterization of the role of distinct plasma cell-free DNA species in age-associated inflammation and frailty. Aging Cell 12, 388-397.

Jylhava, J., Raitanen, J., Marttila, S., Hervonen, A., Jylha, M., Hurme, M., 2014. Identification of a prognostic signature for old-age mortality by integrating genome-wide transcriptomic data with the conventional predictors: the Vitality 90+ Study. BMC Med. Genomics 7, 54

Kelaiditi, E., Cesari, M., Canevelli, M., van Kan, G., Ousset, P., Gillette-Guyonnet, S., Ritz, P., Duveau, F., Soto, M., Provencher, V., Nourhashemi, F., Salvà, A., Robert, P., Andrieu, S., Rolland, Y., Touchon, J., Fitten, J., Vellas, B., IANA/IAGG, 2013. Cognitive frailty: rational and definition from an (I.A.N.A./I.A.G.G.) international consensus group. J. Nutr. Health Aging 17, 726-734

Kim, S., Welsh, D.A., Myers, L., Cherry, K.E., Wyckoff, J., Jazwinski, S.M., 2015 Non-coding genomic regions possessing enhancer and silencer potential are associated with healthy aging and exceptional survival. Oncotarget 6 , 3600-3612

Klungland, A., Bjelland, S., 2007. Oxidative damage to purines in DNA: role of mammalian Ogg1. DNA Repair 6, 481-488.
Lang, P.O., Michel, J.P., Zekry, D., 2009. Frailty syndrome: a transitional state in a dynamic process. Gerontology 55, 539-549.

Li, H., Manwani, B., Leng, S.X., 2011. Frailty, inflammation, and immunity. Aging Dis. 2, 466-473.

Liu, C.K., Lyass, A., Larson, M.G., Massaro, J.M., Wang, N., D’Agostino Sr., R.B., Benjamin, E.J., Murabito, J.M., 2016. Biomarkers of oxidative stress are associated with frailty: the Framingham Offspring Study. Age (Dordr) 38, 1.

Marzetti, E., Lorenzi, M., Antocicco, M., Bonassi, S., Celi, M., Mastropaolo, S., Settanni, S., Valdiglesias, V., Landi, F., Bernabei, R., Onder, G., 2014. Shorter telomeres in peripheral blood mononuclear cells from older persons with sarcopenia: results from an exploratory study. Front Aging Neurosci. 6, 233.

Matteini, A.M., Walston, J.D., Bandeen-Roche, K., Arking, D.E., Allen, R.H., Fried, L.P., Chakravarti, A., Stabler, S.P., Fallin, M.D., 2010. Transcobalamin-II variants, decreased vitamin B12 availability and increased risk of frailty. J. Nutr. Health Aging 14, 73-77.

Migliore, L., Coppede, F., 2002. Genetic and environmental factors in cancer and neurodegenerative diseases. Mutat. Res. 512, 135-153.

Migliore, L., Coppede, F., Fenech, M., Thomas, P., 2011. Association of micronucleus frequency with neurodegenerative diseases. Mutagenesis 26, 85-92.

Mikhelson, V.M., Gamaley, I.A., 2012. Telomere shortening is a sole mechanism of aging in mammals. Curr. Aging Sci. 5, 203-208.

Mitnitski, A.B., Mogilner, A.J., Rockwood, K., 2001. Accumulation of deficits as a proxy measure of aging. Sci. World J. 1, 323-336.

Mladinic, M., Kopjar, N., Milic, M., Dasovic, A.B., Huzak, M., Zeljezic, D., 2010. Genomic instability in a healthy elderly population: a pilot study of possible cytogenetic markers related to ageing. Mutagenesis 25, 455-462

Montesanto, A., Lagani, V., Martino, C., Dato, S., De Rango, F., Berardelli, M., Corsonello, A., Mazzei, B., Mari, V., Lattanzio, F., Conforti, D., Passarino, G., 2010. A novel, population-specific approach to define frailty. Age (Dordr) 32, 385-395.

Moore, A.Z., Biggs, M.L., Matteini, A., O’Connor, A., McGuire, S., Beamer, B.A., Fallin, M.D., Fried, L.P., Walston, J., Chakravarti, A., Arking, D.E., 2010. Polymorphisms in the mitochondrial DNA control region and frailty in older adults. PLoS One 5 e11069.

Namioka, N., Hanyu, H., Hirose, D., Hatanaka, H., Sato, T., Shimizu, S., 2016. Oxidative stress and inflammation are associated with physical frailty in patients with Alzheimer's disease. Geriatr. Gerontol. Int.

Pereira, M.C., Miralles, R., Serra, E., Morros, A., Palacio, J.R., Martinez, P., 2016. Lipid peroxidation in the lymphocyte membrane and protein oxidation in the serum of elderly people. Are they potential markers of frailty and dependence? Preliminary results. Rev. Esp. Geriatr. Gerontol. 51, 25-28.

Perry, G., Nunomura, A., Hirai, K., Zhu, X., Pérez, M., Avila, J., Castellani, R., Atwood, C., Aliev, G., Sayre, L., Takeda, A., Smith, M., 2002. Is oxidative damage the fundamental pathogenic mechanism of Alzheimer's and other neurodegenerative diseases? Free Radic. Biol. Med. 33, 1475-1479

Rao, K.S., 2009. Free radical induced oxidative damage to DNA: relation to brain aging and neurological disorders. Indian J. Biochem. Biophys. 46, 9-15.

Rattan, S.I., 2006. Theories of biological aging: genes, proteins, and free radicals. Free Radic. Res. 40, 1230-1238.

Rattan, S.I., 2012. Biogerontology: from here to where?: the lord cohen medal lecture-2011. Biogerontology 13, 83-91.

Rockwood, K., Song, X., MacKnight, C., Bergman, H., Hogan, D.B., McDowell, I., Mitnitski, A., 2005. A global clinical measure of fitness and frailty in elderly people. CMAJ 173, 489-495

Roland, K.P., Theou, O., Jakobi, J.M., Swan, L., Jones, G.R., 2014. How do community physical and occupational therapists classify frailty? A pilot study. J. Frailty Aging 3, 247-250

Saum, K.U., Dieffenbach, A.K., Muezzinler, A., Muller, H., Holleczek, B., Stegmaier, C., Butterbach, K., Schick, M., Canzian, F., Stammer, H., Boukamp, P., Hauer, K. Brenner, $H_{.}$2014. Frailty and telomere length: cross-sectional analysis in 3537 older adults from the ESTHER cohort. Exp. Gerontol. 58, 250-255.

Saum, K.U., Dieffenbach, A.K., Jansen, E.H., Schottker, B., Holleczek, B., Hauer, K., Brenner, H., 2015. Association between oxidative stress and frailty in an elderly german population: results from the ESTHER cohort study. Gerontology 61, 407-415

Sedelnikova, O.A., Horikawa, I., Zimonjic, D.B., Popescu, N.C., Bonner, W.M., Barrett, J.C., 2004. Senescing human cells and ageing mice accumulate DNA lesions with unrepairable double-strand breaks. Nat. Cell Biol. 6, 168-170.

Sedelnikova, O.A., Horikawa, I., Redon, C., Nakamura, A., Zimonjic, D.B., Popescu, N.C., Bonner, W.M., 2008. Delayed kinetics of DNA double-strand break processing in normal and pathological aging. Aging Cell 7, 89-100.

Serviddio, G., Romano, A.D., Greco, A., Rollo, T., Bellanti, F., Altomare, E., Vendemiale, G., 2009. Frailty syndrome is associated with altered circulating redox balance and increased markers of oxidative stress. Int. J. Immunopathol. Pharmacol. 22, 819-827.

Sieber, C.C., 2017. Frailty - from concept to clinical practice. Exp. Gerontol. 87. $160-167$.

Strasser, B., Becker, K., Fuchs, D., Gostner, J.M., 2017. Kynurenine pathway metabolism and immune activation: peripheral measurements in psychiatric and co-morbid conditions. Neuropharmacology 112, 286-296.

Theou, O., Brothers, T.D., Mitnitski, A., Rockwood, K., 2013. Operationalization of frailty using eight commonly used scales and comparison of their ability to predict all-cause mortality. J. Am. Geriatr. Soc. 61, 1537-1551.

Theou, O., Cann, L., Blodgett, J., Wallace, L.M., Brothers, T.D., Rockwood, K., 2015. Modifications to the frailty phenotype criteria Systematic review of the current 
literature and investigation of 262 frailty phenotypes in the Survey of Health, Ageing, and Retirement in Europe. Ageing Res. Rev. 21, 78-94.

Thomas, P., Fenech, M., 2007. A review of genome mutation and Alzheimer's disease. Mutagenesis 22, 15-33.

Topinková, E., 2008. Aging, disability and frailty. Ann. Nutr. Metab. 52 (Suppl. 1), $6-11$.

Valdiglesias, V., Pasaro, E., Mendez, J., Laffon, B., 2011. Assays to determine DNA repair ability. J. Toxicol. Environ. Health A 74, 1094-1109.

Valdiglesias, V., Bonassi, S., Dell'Armi, V., Settanni, S., Celi, M., Mastropaolo, S., Antocicco, M., Fini, M., Onder, G., 2015. Micronucleus frequency in peripheral blood lymphocytes and frailty status in elderly: a lack of association with clinical features. Mutat. Res. 780, 47-54.

Walston, J., 2004. Frailty?the search for underlying causes. Sci. Aging Knowledge Environ. 2004 (pe4)

Wang, C., Jurk, D., Maddick, M., Nelson, G., Martin-Ruiz, C., von Zglinicki, T., 2009. DNA damage response and cellular senescence in tissues of aging mice. Aging Cell 8, 311-323.
Woo, J., Tang, N.L., Suen, E., Leung, J.C., Leung, P.C., 2008. Telomeres and frailty. Mech. Ageing Dev. 129, 642-648.

Woods, J.A., Wilund, K.R., Martin, S.A., Kistler, B.M., 2012. Exercise, inflammation and aging. Aging Dis. 3, 130-140.

Woods, A.J., Cohen, R.A., Pahor, M., 2013. Cognitive frailty: frontiers and challenges. J. Nutr. Health Aging 17, 741-743.

Wu, I.C., Shiesh, S.C., Kuo, P.H., Lin, X.Z., 2009. High oxidative stress is correlated with frailty in elderly chinese. J. Am. Geriatr. Soc. 57, 1666-1671.

Yu, R., Tang, N., Leung, J., Woo, J., 2015. Telomere length is not associated with frailty in older Chinese elderly: cross-sectional and longitudinal analysis. Mech. Ageing Dev. 152, 74-79.

Zaslavsky, O., Cochrane, B.B., Thompson, H.J., Woods, N.F., Herting, J.R., LaCroix, A., 2013. Frailty: a review of the first decade of research. Biol. Res. Nurs. 15, $422-432$ 